\title{
Vacuum polarization radiative correction to the parity violating electron scattering on heavy nuclei
}

\author{
A. I. Milstein* \\ Budker Institute of Nuclear Physics, 630090 Novosibirsk, Russia \\ O. P. Sushkov \\ School of Physics, University of New South Wales, Sydney 2052, Australia
}

(Dated: November 15, 2018)

\begin{abstract}
The effect of vacuum polarization on the parity violating asymmetry in the elastic electron-nucleus scattering is considered. Calculations are performed in the high-energy approximation with an exact account for the electric field of the nucleus. It is shown that the radiative correction to the parity violating asymmetry is logarithmically enhanced and the value of the correction is about $-1 \%$.
\end{abstract}

PACS numbers: $24.80 .+\mathrm{y}, 25.30 . \mathrm{Bf}, 21.10 . \mathrm{Gv}$

Experimental investigations of the proton $\rho_{p}(r)$ and neutron $\rho_{n}(r)$ densities in heavy nuclei are very important for testing of various nuclear models. The densities are also important for many applications like, for example, precise calculations of atomic parity nonconservation. While the charge distribution (proton density) is known pretty well mainly due to data on the elastic electron scattering, the determination of the neutron density with a good accuracy is a very complicated problem [1]. It was suggested by Donnelly et al 2] to use parity violation (PV) in the electronnucleus scattering to determine the neutron distribution. The idea behind this suggestion is that the mediator of the weak interaction, Z-boson, interacts mainly with neutrons. The parity violating asymmetry is defined as

$$
\mathcal{A}_{P V}=\frac{d \sigma_{+} / d \Omega-d \sigma_{-} / d \Omega}{d \sigma_{+} / d \Omega+d \sigma_{-} / d \Omega}
$$

where $d \sigma_{+} / d \Omega$ and $d \sigma_{-} / d \Omega$ are cross sections for scattering of right-handed and left-handed electrons, respectively. The asymmetry can be measured experimentally and in order to determine the neutron distribution one needs to calculate the asymmetry at a given distribution. The scattering potential is of the form

$$
V(r)=V_{C}(r)+\gamma_{5} A(r), \quad A(r)=\frac{G_{F}}{2 \sqrt{2}} \varrho_{W}(r) .
$$

Here $V_{C}(r)$ is the Coulomb potential of the nucleus, $G_{F}=1.16639 \times 10^{-5} \mathrm{GeV}^{-2}$ is the Fermi constant, $\gamma_{5}$ is the Dirac matrix, and $\varrho_{W}(r)$ is the density of the weak charge

$$
\varrho_{W}(r)=-N \varrho_{n}(r)+\left(1-4 \sin ^{2} \theta_{W}\right) Z \varrho_{p}(r),
$$

where $\theta_{W}$ is the Weinberg angle $\left(\sin ^{2} \theta_{W} \approx 0.23\right), N$ and $Z$ are numbers of protons and neutrons, respectively. The densities are normalized as $\int d \boldsymbol{r} \varrho_{p, n}(r)=1$. In this work we consider a high energy scattering, so the electron mass can be neglected compared to energy. In this limit the cross sections $d \sigma_{+} / d \Omega$ and $d \sigma_{-} / d \Omega$ correspond to scattering in potentials $V_{C}(r)+A(r)$ and $V_{C}(r)-A(r)$, respectively. The PV asymmetry is due to interference between the Coulomb amplitude and the amplitude caused by the axial potential $A(r)$. Since neutrons dominate in the weak-charge density $\varrho_{W}(r)$ (3), the asymmetry $\mathcal{A}_{P V}$ is very sensitive to the shape of $\varrho_{n}(r)$. Though the value of $\mathcal{A}_{P V}$ is very small and its measurement is a very complex problem, there is a proposal $[\underline{3}]$ for the corresponding experiment.

Detailed theoretical investigation of the asymmetry $\mathcal{A}_{P V}$ has been performed by Horowits [4], Vretenar et al 5 ], and by Horowits et al [6]. It has been shown that $\mathcal{A}_{P V}$ is very sensitive to the small difference between $\varrho_{p}(r)$ and $\varrho_{n}(r)$. It has been also shown that the Coulomb distortions of the electron wave functions significantly modify the asymmetry as compared to that obtained in the plane-wave approximation. In this case radiative corrections can be enhanced. The corrections have not been considered before. In the present work we calculate the vacuum polarization radiative correction to $\mathcal{A}_{P V}$. We found that similar to radiative corrections to atomic parity violation [7, 8] the correction we consider here is logarithmically enhanced. The logarithm is $\ln \left(\lambda_{C} / r_{0}\right)$, where $r_{0} \approx 1.5 Z^{1 / 3} \mathrm{fm}$ is the nuclear radius and $\lambda_{C}$ is the electron Compton wavelength.

\footnotetext{
*Email A.I.Milstein@inp.nsk.su

†Email sushkov@phys.unsw.edu.au
} 
It is well known that in the leading $Z \alpha$ approximation $(\alpha=1 / 137$ is the fine-structure constant), the vacuum polarization results in the Uehling potential [9]. At $r_{0} \ll r \ll \lambda_{C}$, this potential is of the form $V(r) \approx 2 Z \alpha^{2}\left[\ln \left(r / \lambda_{C}\right)+\right.$ $C+5 / 6] /(3 \pi r)$, where $C \approx 0.577$ is the Euler constant. Account of corrections higher in $Z \alpha$ leads to a modification of the constant: $C \rightarrow C+0.092 Z^{2} \alpha^{2}+\ldots$, see Ref. [10]. This correction is small and can be neglected even for $Z \alpha \sim 1$. For our purpose it is necessary to calculate the vacuum polarization potential at $r \sim r_{0}$ where the presented above simple logarithmic approximation is not sufficient. Nevertheless the conclusion about smallness of the $Z \alpha$-corrections to the Uehling potential remains valid at $r \sim r_{0}$ and hence we will use the Uehling potential in our calculations. In Refs. [4, 5, 6], the scattering amplitude has been obtained as a result of summation over partial waves. We do not use a partial wave analysis, instead we use the high-energy small-angle approximation. The scattering amplitude in this approximation reads

$$
\begin{aligned}
& f=-\frac{i p}{2 \pi} \int d \boldsymbol{\rho} \exp [-i \boldsymbol{q} \boldsymbol{\rho}]\{\exp [-i \chi(\rho)]-1\}, \\
& \chi(\rho)=\int_{-\infty}^{\infty} d z V(r), r=\sqrt{z^{2}+\rho^{2}}
\end{aligned}
$$

where $\boldsymbol{q}=\boldsymbol{p}_{2}-\boldsymbol{p}_{1}$ is the momentum transfer, z-axis is directed along the vector $\boldsymbol{p}=\left(\boldsymbol{p}_{2}+\boldsymbol{p}_{1}\right) / 2$, and $\boldsymbol{\rho}$ is a two-dimensional vector orthogonal to $\boldsymbol{p}$. Here $\boldsymbol{p}_{1}$ and $\boldsymbol{p}_{2}$ is the initial and the final electron momentum, respectively. Validity of Eq. (4) has been discussed in numerous works, see book [1] and references therein. At first, this formula has been derived using wave functions in the eikonal approximation. Then, Olsen et al [12] have demonstrated that the range of validity is wider: the semiclassical approximation is sufficient to justify Eq. (4) even if the eikonal approximation is not valid. Corrections to Eq. (4) are considered in Ref. [13], see also Ref. [14]. For high-energy small-angle scattering the semiclassical approximation and hence formula (4) is well justified and we will use it. From Eqs. (11) and (4), we obtain

$$
\begin{aligned}
& \mathcal{A}_{P V}=2 \frac{\operatorname{Re}\left[f_{C}^{*} f_{W}\right]}{\left|f_{C}\right|^{2}} \\
& f_{C}=-\frac{i p}{2 \pi} \int d \boldsymbol{\rho} \exp [-i \boldsymbol{q} \boldsymbol{\rho}]\left\{\exp \left[-i \chi_{C}(\rho)\right]-1\right\} \\
& f_{W}=-\frac{p G_{F}}{4 \sqrt{2} \pi} \int d \boldsymbol{\rho} \exp \left[-i \boldsymbol{q} \boldsymbol{\rho}-i \chi_{C}(\rho)\right] \int_{-\infty}^{\infty} d z \varrho_{W}(r) \\
& \chi_{C}(\rho)=\int_{-\infty}^{\infty} d z V_{C}(r),
\end{aligned}
$$

To simplify further numerical integrations, it is convenient to express $\chi_{C}(\rho)$ in terms of $\varrho_{p}$,

$$
\begin{aligned}
& \chi(\rho)=2 Z \alpha \Phi(\rho), \\
& \Phi(\rho)=\ln (\rho / L)+4 \pi \int_{\rho}^{\infty} d r r \varrho_{p}(r)\left[r \ln \left(\frac{r+\sqrt{r^{2}-\rho^{2}}}{\rho}\right)-\sqrt{r^{2}-\rho^{2}}\right] .
\end{aligned}
$$

Here $L \gg r_{0}$ is an arbitrary constant, the cross sections and the asymmetry are independent of the constant. The function $\Phi(\rho)$ has the following properties:

$$
\Phi(\rho)=\left\{\begin{array}{c}
4 \pi \int_{0}^{\infty} d r r^{2} \varrho_{p}(r) \ln (2 r / L)-1, \quad \text { at } \rho \rightarrow 0 \\
\ln (\rho / L), \quad \text { at } \rho \gg r_{0} .
\end{array}\right.
$$

Taking in Eq. (5) the integral over the angle of the vector $\rho$ and using Eq. (6), we obtain

$$
\begin{aligned}
& f_{C}=\frac{2 Z \alpha p}{q^{2}}\left\{4 \pi \int_{0}^{\infty} d \rho \rho J_{0}(q \rho) \exp [-2 i Z \alpha \Phi(\rho)] \int_{\rho}^{\infty} \frac{d r r \varrho_{p}(r)}{\sqrt{r^{2}-\rho^{2}}}\right. \\
& \left.-2 i Z \alpha \int_{0}^{\infty} \frac{d \rho}{\rho} J_{0}(q \rho) \exp [-2 i Z \alpha \Phi(\rho)] \tilde{\Phi}^{2}(\rho)\right\}, \\
& f_{W}=-\frac{p G_{F}}{\sqrt{2}} \int_{0}^{\infty} d \rho \rho J_{0}(q \rho) \exp [-2 i Z \alpha \Phi(\rho)] \int_{\rho}^{\infty} \frac{d r r \varrho_{W}(r)}{\sqrt{r^{2}-\rho^{2}}}
\end{aligned}
$$

where

$$
\tilde{\Phi}(\rho)=\rho \frac{\partial \Phi(\rho)}{\partial \rho}=1-4 \pi \int_{\rho}^{\infty} d r r \varrho_{p}(r) \sqrt{r^{2}-\rho^{2}}
$$


and $J_{0}(x)$ is the Bessel function. Amplitudes in form (8) are very convenient for numerical integration due to fast convergence of the integrals. In the Born approximation (plane wave approximation) the amplitudes and the asymmetry are

$$
\begin{aligned}
& f_{C}^{B}=\frac{2 Z \alpha p}{q^{2}} F_{p}(q) \quad, \quad f_{W}^{B}=-\frac{p G_{F} F_{W}(q)}{4 \pi \sqrt{2}}, \\
& \mathcal{A}_{P V}^{B}=-\frac{q^{2} G_{F} F_{W}(q)}{4 \pi \sqrt{2} Z \alpha F_{p}(q)}=\frac{q^{2} G_{F}}{4 \pi \sqrt{2} \alpha}\left[\frac{N F_{n}(q)}{Z F_{p}(q)}+4 \sin ^{2} \theta_{W}-1\right] .
\end{aligned}
$$

Here $F_{p}(q), F_{n}(q)$, and $F_{W}(q)$ are Fourier transforms (form factors) of corresponding densities. If neutron and proton distributions coincide, $\varrho_{n}(r)=\varrho_{p}(r)$, then the Born approximation asymmetry (9) is given by

$$
\mathcal{A}_{0}=\frac{G_{F} q^{2}}{4 \pi \sqrt{2} \alpha}\left[\frac{N}{Z}+4 \sin ^{2} \theta_{W}-1\right] .
$$

We will use this asymmetry as a reference point. Figure 1 shows dependence of the ratio $\mathcal{A}_{P V} / \mathcal{A}_{0}$ on the momentum transfer $q$ for $\mathrm{Pb}(\mathrm{Z}=82)$ at $\varrho_{n}(r)=\varrho_{p}(r)$ (solid curve) and at $\varrho_{n}(r)=0.95^{3} \varrho_{p}(0.95 r)$ (dashed curve). For $\varrho_{p}(r)$ we

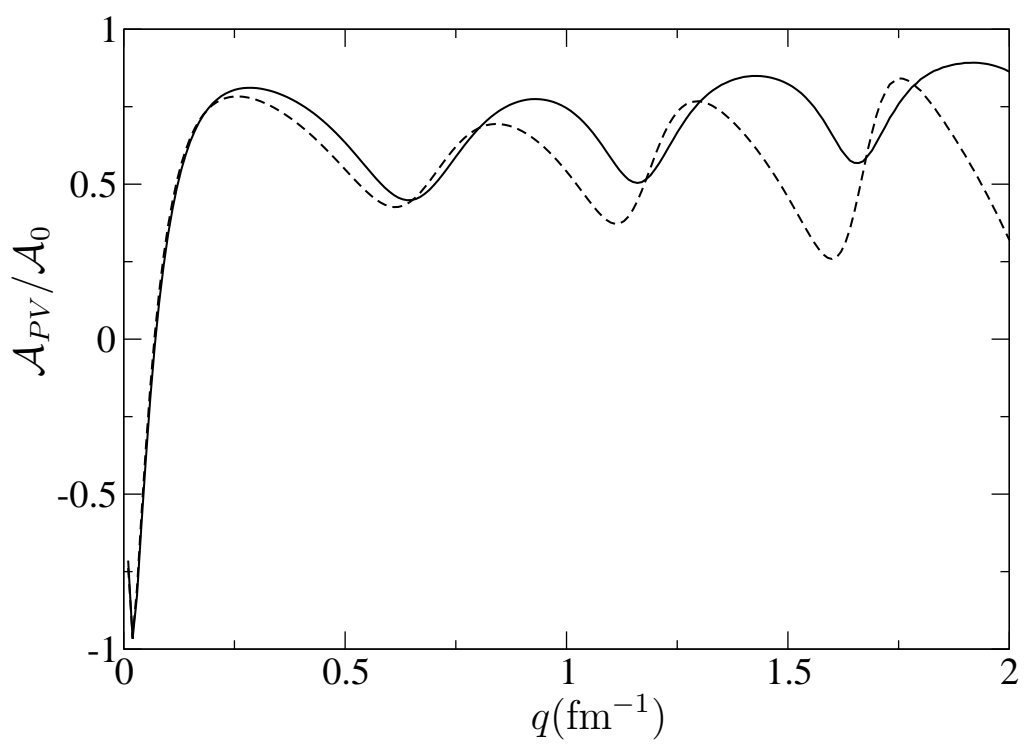

FIG. 1: Dependence of the ratio $\mathcal{A}_{P V} / \mathcal{A}_{0}$ for $\mathrm{Pb}(Z=82)$ on the momentum transfer $q$. The solid line corresponds to $\varrho_{n}(r)=$ $\varrho_{p}(r)$ and the dashed line corresponds to $\varrho_{n}(r)=\lambda^{3} \varrho_{p}(\lambda r)$ with $\lambda=0.95$. The reference Born approximation asymmetry $\mathcal{A}_{0}$ is given by Eq. (10)

use the three parameter Fermi charge density [4]:

$$
\begin{aligned}
& \varrho_{p}(r)=\varrho_{0} \frac{1+w\left(r / r_{0}\right)^{2}}{1+\exp \left[\left(r-r_{0}\right) / a\right]} \quad, \\
& r_{0}=6.4 \mathrm{fm}, \quad a=0.54 \mathrm{fm}, \quad w=0.32, \quad \varrho_{0}=6.95 \times 10^{-4} \mathrm{fm}^{-3}
\end{aligned}
$$

Plots in Fig. 1]are in excellent agreement with results of the partial wave analysis 4]. The figure clearly demonstrates high sensitivity to the difference between $\varrho_{n}(r)$ and $\varrho_{p}(r)$ as well as importance of the Coulomb distortion. The distortion is especially important at small $q$. At $q \ll 1 / r_{0}$ the Coulomb and the weak amplitudes are of the form

$$
\begin{aligned}
& f_{C}=\frac{2 Z \alpha p}{q^{2}}\left(\frac{q L}{2}\right)^{2 i Z \alpha} \frac{\Gamma(1-i Z \alpha)}{\Gamma(1+i Z \alpha)}, \\
& f_{W}=-\frac{p G_{F}}{\sqrt{2}} \int_{0}^{\infty} d \rho \rho \exp [-2 i Z \alpha \Phi(\rho)] \int_{\rho}^{\infty} \frac{d r r \varrho_{W}(r)}{\sqrt{r^{2}-\rho^{2}}}
\end{aligned}
$$


So at $q \rightarrow 0$ the weak amplitude $f_{W}$ is independent of $q$ while phase of the Coulomb amplitude $f_{C}$ strongly depends on $q$. This explains oscillations of $\mathcal{A}_{P V}$ at $q \rightarrow 0$.

Let us consider now influence of vacuum polarization on the parity violating asymmetry. The charge density $\varrho_{v p}(r)$ induced by polarization of the electron-positron vacuum and expressed in units of the elementary charge $|e|$ is of the form, see e.g. the review paper 15$]$

$$
\begin{aligned}
& \varrho_{v p}(r)=\frac{Z \alpha}{3 \pi} \int_{1}^{\infty} d \tau \sqrt{\frac{\tau-1}{\tau}} \frac{(\tau+1 / 2)}{\tau^{2}} \\
& \times\left\{\varrho_{p}(r)-\frac{m^{2} \tau}{\pi} \int d \boldsymbol{R} \frac{\exp (-2 m \sqrt{\tau}|\boldsymbol{r}-\boldsymbol{R}|)}{|\boldsymbol{r}-\boldsymbol{R}|} \varrho_{p}(R)\right\} .
\end{aligned}
$$

This charge density corresponds to the Uehling potential [9]. For small distances, $r \ll \lambda_{C}$, expression (13) can be transformed to

$$
\begin{aligned}
& \varrho_{v p}(r) \rightarrow \frac{2 Z \alpha}{3 \pi}\left\{\left[\ln \left(\lambda_{C} / r\right)-C-5 / 6\right)\right] \varrho_{p}(r) \\
& +\frac{1}{r} \int_{0}^{r} d R \varrho_{p}(R)-\frac{1}{2} \int_{0}^{\infty} d R \frac{\varrho_{p}(R)}{r+R} \\
& \left.-\frac{1}{2} \int_{0}^{2 r} d R \frac{\varrho_{p}(R)-\varrho_{p}(r)}{|R-r|}-\frac{1}{2} \int_{2 r}^{\infty} d R \frac{\varrho_{p}(R)}{R-r}\right\} .
\end{aligned}
$$

Note that Eq. (14) is not singular at $r=0$, all the divergent terms are canceled out. Outside of nucleus, at $r \gg r_{0}$, Eq.(13) is equivalent to the well known formula for the induced charge density of a point-like nucleus

$$
\varrho_{v p}(r)=-\frac{2 Z \alpha m^{2}}{3 \pi^{2} r} \int_{1}^{\infty} d x \sqrt{x^{2}-1}\left(1+\frac{1}{2 x^{2}}\right) \exp (-2 m r x)
$$

At $\lambda_{C} \gg r \gg r_{0}$ it gives

$$
\varrho_{v p}(r)=-\frac{Z \alpha}{6 \pi^{2} r^{3}}
$$

A direct numerical integration of (13) is straightforward. In Fig. 2 we show by solid line the vacuum polarization charge density $\varrho_{v p}(r)$ for $\mathrm{Pb}$. For comparison the dashed line presents the proton density (11). We remind that $\varrho_{p}(r)$

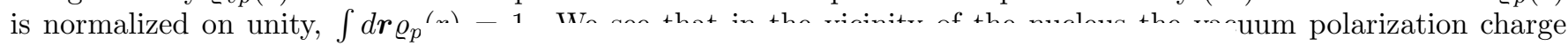

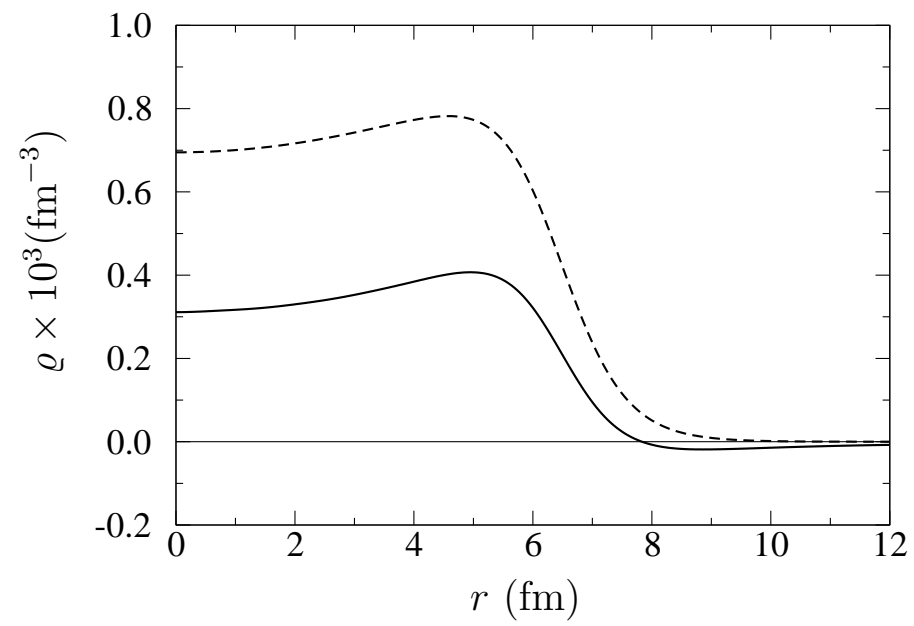

FIG. 2: Solid line: the vacuum polarization charge density $\varrho_{v p}(r)$ for Pb in units $10^{-3}|e| / \mathrm{fm}^{3}$. Dashed line: the proton density $\varrho_{p}(r)$ in units $10^{-3} \mathrm{fm}^{-3}$. The proton density is normalized to unity.

density is practically proportional to the proton density. With logarithmic accuracy this fact immediately follows from Eq. (14)

$$
\varrho_{v p}(r) \sim \frac{2 Z \alpha}{3 \pi} \ln \left(\frac{\lambda_{C}}{r_{0}}\right) \varrho_{p}(r)
$$


It is interesting that inside a sphere of radius $8 \mathrm{fm}$ the vacuum polarization charge is rather big and equals to $0.47|e|$. So one can say that the electric charge of the $\mathrm{Pb}$ nucleus is 82.47 . Certainly the excess charge 0.47 is compensated by the negative charge -0.47 distributed between $r_{0}$ and $\lambda_{C}$, see Eq. (16). Using Eqs. (14), (15) and (8), it is easy to calculate the asymmetry $\mathcal{A}_{\text {tot }}$ that account for the effect of vacuum polarization. One should replace $\varrho_{p}(r)$ in (8) to $\varrho_{p}(r)+\frac{1}{Z} \varrho_{v p}(r)$. Note that it is not necessary to account for the effect of vacuum polarization on $\varrho_{W}(r)$ because the corresponding correction contains the additional suppression factor $1-4 \sin ^{2} \theta_{W}$ in the electron- $Z^{0}$ vector vertex in the electron loop. With account of the radiative correction the parity violating asymmetry is reduced, $\mathcal{A}_{P V} \rightarrow \mathcal{A}_{P V}(1+\Delta)$. Value of the vacuum polarization radiative correction $\Delta$ in per cent is plotted in Fig. 3 versus the momentum transfer $q$. In the interesting range of momenta, $q=0.2 \div 2 \mathrm{fm}^{-1}$, the radiative correction is about

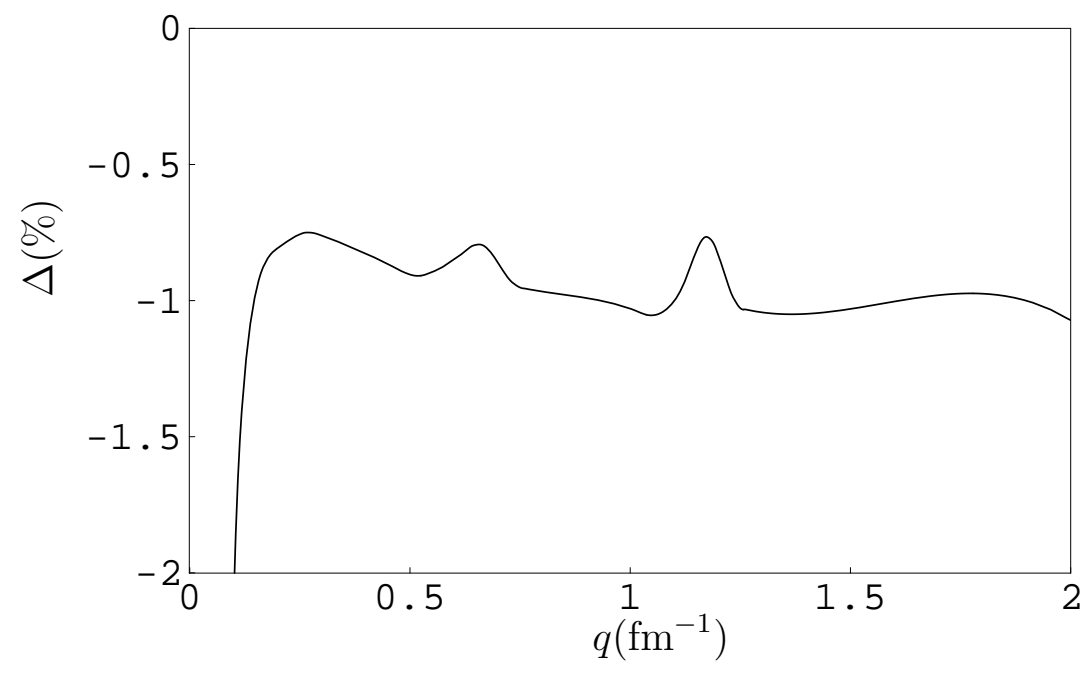

FIG. 3: The vacuum polarization radiative correction to the parity violation asymmetry (\%) versus the momentum transfer.

-1\%. A similar value one can expect in the Born approximation. In this approximation only the denominator $F_{p}(q)$ in Eq. (9) is influenced by the correction. Therefore, due to (17) the radiative correction is

$$
\Delta^{(B o r n)} \sim-\frac{2 \alpha}{3 \pi} \ln \left(\frac{\lambda_{C}}{r_{0}}\right) \approx-0.7 \%
$$

We have considered the vacuum polarization correction to the parity violating asymmetry. There are also electron self-energy and vertex corrections corresponding to radiation and absorption of virtual photons, and to radiation of real photons. It is well known that these corrections to the scattering amplitude are very big due to double logarithms. For example the vertex correction to the Born scattering cross section is [16]

$$
\Delta_{\sigma}=\exp \left(-\frac{2 \alpha}{\pi} \ln \frac{-q^{2}}{m^{2}} \ln \frac{E}{\Omega}\right)-1 .
$$

Here $q$ is the momentum transfer, $E$ and $m$ are the electron energy and mass, respectively, and $\Omega$ is resolution with respect to bremsstrahlung. For conditions of proposal [3] $(E=850 \mathrm{MeV}, q=90 \mathrm{MeV}, \Omega=4 \mathrm{MeV})$ the correction is $-23 \%$ (!). However, in the Born approximation the vertex correction is canceled out in the parity violating asymmetry $\mathcal{A}_{P V}$. Certainly the Born approximation is not sufficient to describe electron scattering on $\mathrm{Pb}$, and a calculation of the vertex correction to $\mathcal{A}_{P V}$ in this case is an open and a very hard problem.

Concluding we have calculated the effect of vacuum polarization on the parity violating asymmetry in the elastic electron-nucleus scattering. The vacuum polarization radiative correction is logarithmically enhanced and the value of the correction is about $-1 \%$. 
A.I.M. gratefully acknowledges the School of Physics at the University of New South Wales for warm hospitality and financial support during a visit. The work was also supported by RFBR Grant No. 03-02-16510.

[1] C.J. Batty, E. Friedman, H.J. Gils, and H. Rebel, Adv. Nucl. Phys. 19, 1 (1989).

[2] T.W. Donnelly, J Dubach, and Ingo Sick, Nucl. Phys. A503, 589 (1989).

[3] P.A. Souder, R.W. Michaelse, and G.M. Urciuoli,Jefferson Lab Proposal E00003; This proposal is on the web at www.jlab.org/ rom/pbpro.ps ; www.jlab.org/ rom/pbupdate.ps.

[4] C.J. Horowitz, Phys. Rev. C 57, 3430 (1998).

[5] D. Vretenar, P. Finelli, A. Ventura, G.A. Lalazissis, and P.Ring, Phys. Rev. C 61, 064307 (2000).

[6] C.J. Horowitz, S.J. Pollock, P.A.Souder, R.Michaels, Phys. Rev. C 63, 025501 (2001).

[7] A. I. Milstein and O. P. Sushkov, Phys. Rev. A 66, 022108 (2002).

[8] A. I. Milstein, O. P. Sushkov and I. S. Terekhov, Phys. Rev. Lett., 89, 283003 (2002); Phys. Rev. A 67, 062103 (2003).

[9] E. A. Uehling, Phys. Rev. 48, 55 (1935).

[10] A. I. Milstein and V. M. Strakhovenko, Zh. Eksp. Teor. Fiz. 84, 1247 (1983) [Sov. Phys. JETP 57, 722 (1983)].

[11] H. Überall, Electron scattering from compex nuclei (Academic Press, NY and London, 1971).

[12] H. Olsen, L.C. Maximon, and H. Wergeland, Phys. Rev., 106, 27 (1957).

[13] R.N. Lee, A.I. Milstein, and V.M. Strakhovenko, Zh. Eksp. Teor. Fiz. 117, 75 (2000) [JETP 90, 66 (2000)].

[14] A.I. Akhiezer, V.F. Boldyshev, and N.F. Shul'ga, Teor. Mat. Fiz. 23, 11 (1975).

[15] E. Borie, and G.A. Rinker, Rev. Mod. Phys. 54, 67 (1982).

[16] V. B. Berestetskii, E. M. Lifshitz, L. P. Pitaevskii, Relativistic quantum theory, Pergamon Press, Oxford, 1982. 